ate a fatty diet during lactation had pups that were fatter, had higher insulin levels and were less sensitive to insulin than the offspring of mothers that ate a normal diet. In the fat pups, fewer fibres from specific neurons branched into regions of the brain's hypothalamus that regulate energy metabolism.

This circuitry is established in mice shortly after birth, but in humans it develops during the last trimester of pregnancy. The authors suggest that a mother's diet during this period could have long-term health effects for the child.

Cell http://doi.org/q7k (2014)

\section{ENGINEERING \\ Phone device detects mercury}

A smartphone attachment can detect low levels of mercury in water samples, opening the door to on-site, low-cost environmental monitoring. Inorganic mercury is harmful to the kidneys, and can be converted by bacteria into its neurotoxic, organic forms. The device (pictured), developed by Aydogan Ozcan and his colleagues at the University of California, Los Angeles, can measure inorganic mercury at levels of 3.5 parts per billion (p.p.b.) - good enough to detect the maximum acceptable level of 6 p.p.b. advised by the World Health Organization. The attachment shines green and red light through tiny test tubes, which contain the water sample and a few reagents. The mobile phone's camera detects the light, which shifts towards green wavelengths if mercury is present. $A$

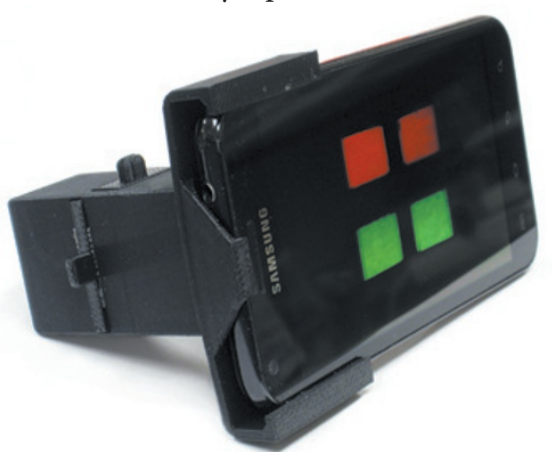

custom-made app provides the measurement.

The researchers tested their device by creating a mercurycontamination map of 50 locations in California. ACS Nano http://doi.org/q6n (2014)

IMMUNOLOGY

\section{Poor diet boosts innate immunity}

Vitamin A deficiency enhances the immune system's response to parasitic worm infections in mice.

Malnutrition typically impairs the body's ability to fight infection. But Yasmine Belkaid at the US National Institutes of Health in Bethesda, Maryland, and her team found that depriving mice of vitamin A boosts an arm of the immune system that protects the body's barriers, such as the gut. Animals lacking this vitamin had a much higher level of ILC2 cells - immune cells that are active in barrier defence — in the gut than mice on a normal diet, and were better able to fend off infection by a nematode worm.

Vitamin A deficiency is common in areas where worm infection is also prevalent. The findings suggest a way that the immune system has adapted to promote survival even in the face of malnutrition.

Science 343, 432-437 (2014)

\section{PUBLIC HEALTH}

\section{Mutations toughen up tuberculosis}

A genomic analysis of the tuberculosis bacterium in a Russian population reveals that the microbe is not only evolving resistance to multiple drugs, but also retaining its ability to survive and spread.

Francis Drobniewski at Queen Mary University of London and his colleagues sequenced the genomes of 1,000 Mycobacterium tuberculosis isolates from people in western Russia.

COMMUNITY CHOICE

The most viewed papers in science

\title{
Dogs domesticated before farming
}

\section{HIGHLY READ \\ on plosgenetics.org 23 Dec-22 Jan}

Dogs became companions for humans long before the advent of agriculture, according to a genome-sequencing study. A team led by Robert Wayne at the University of California, Los Angeles, and John Novembre now at the University of Chicago, Illinois, analysed the genomes of three wolves (Canis lupus) from regions where dogs are thought to have first been domesticated. The authors also studied the genomes of two dog breeds, including Australian dingoes (pictured), and of a golden jackal. The researchers

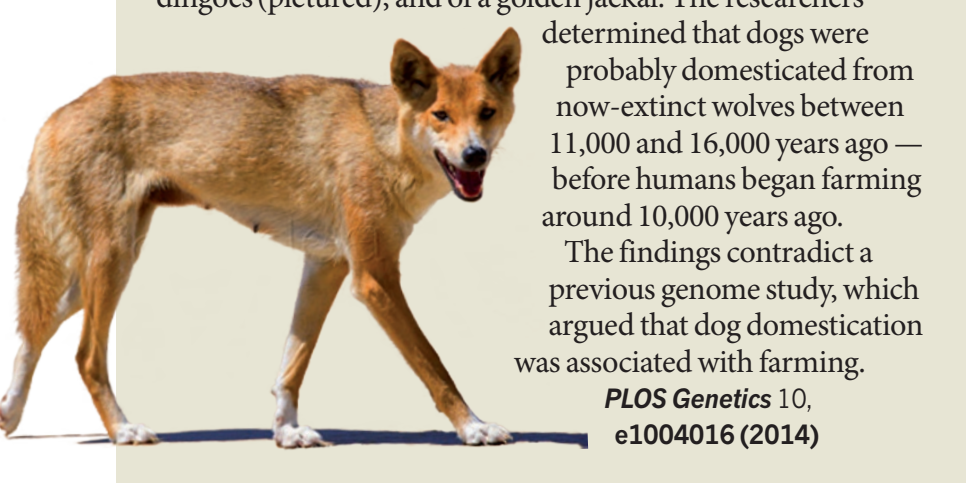

Two-thirds of the isolates belonged to a lineage that first emerged in Asia and is prone to developing drug resistance. More than $60 \%$ of the isolates had drug-resistance mutations. Such mutations typically hinder bacteria's ability to spread, but the team found new 'compensatory' mutations that might maintain transmissibility in more than 400 isolates with resistance to the antibiotic rifampicin.

The findings suggest that biological factors, and not just weak public-health measures, are behind the high incidence of tuberculosis in Russia.

Nature Genetics http://dx.doi. org/10.1038/ng.2878 (2014)

\section{Hot solar cells make more power}

A photovoltaic device that converts sunlight into heat to generate power has achieved greater efficiency than previous such devices, thanks to the design of nanomaterials in the light-absorbing layer.

Thermophotovoltaics contain a layer that absorbs a wider spectrum of wavelengths than conventional solar cells. This layer radiates heat that is used to generate electricity. Evelyn Wang and her team at the Massachusetts Institute of Technology in Cambridge designed their absorberemitter material by growing an array of carbon nanotubes, which turn light into heat, onto a layer of photonic crystals, which they engineered to emit energy of the optimum levels for power generation.

The researchers' device reached an energy conversion efficiency of $3.2 \%$, three times greater than in previous experiments. The authors say that with further improvements, efficiency could exceed $20 \%$.

Nature Nanotech. http://doi.org/ q6j (2014)

\section{$\rightarrow$ NATURE.COM}

For the latest research published by Naturevisit:

www.nature.com/latestresearch 